\title{
RAYMUNDO RAMÍREZ DELGADILLO
}

\author{
(17 DE MARZO DE 1963 - 22 DE FEBRERO DE 2011)
}

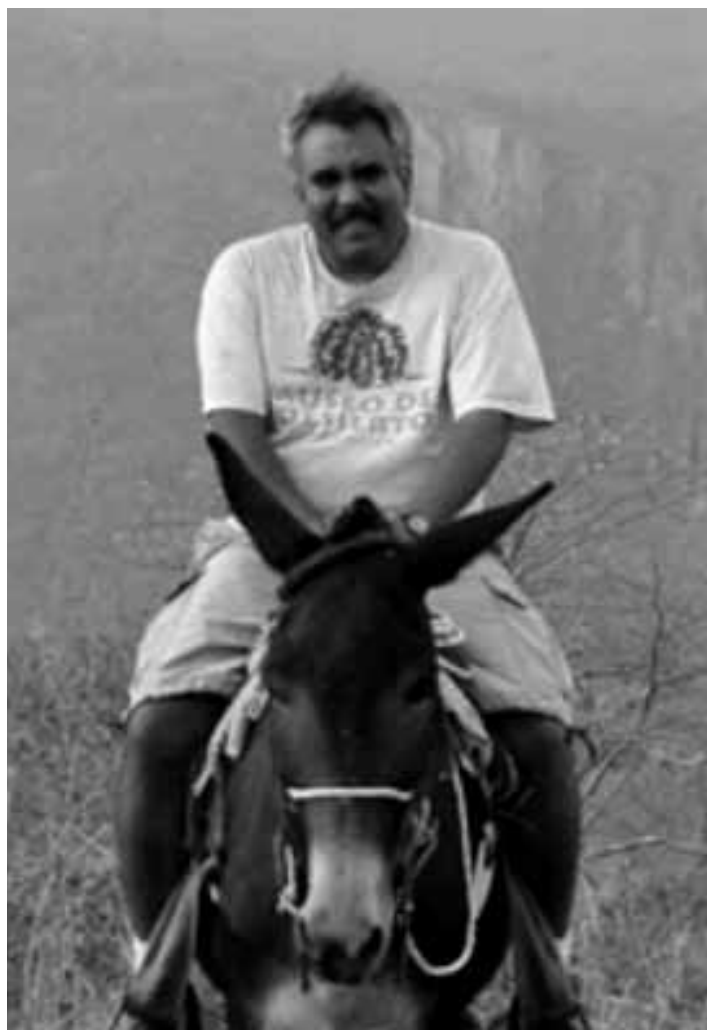

C on tristeza, el herbario Luz María Villarreal de Puga del Instituto de Botánica de la Universidad de Guadalajara (IBUG) informa a la comunidad botánica el sensible fallecimiento de su curador Raymundo Ramírez Delgadillo. Víctima de cáncer, Ray murió el martes 22 de febrero a los 47 años de edad. Le sobreviven sus padres María de Jesús y Margarito. También lo extrañarán sus hermanos Juan, Berenice, Elizabeth, Guillermo, Cristina, Javier, Sergio, Rocío, Mario y Ana. Lo recordaremos todos.

Raymundo nació en Guadalajara, Jalisco, el 17 de marzo de 1963. Fue el primer hijo de una familia de 11 hermanos. Sus estudios de educación básica los realizó en Guadalajara. De igual forma, su adiestramiento profesional lo llevó a cabo en la Facultad de Agricultura de la Universidad de Guadalajara, de septiembre de 1983 a junio de 1988. Su pasión por las plantas surgió desde su infancia, durante las continuas visitas de fines de semana y vacaciones a El Salto, Jalisco, tierra natal de sus padres. Así lo muestra su primera publicación, dedicada a las cactáceas y suculentas de esa localidad. Su interés por la botánica y su habilidad con el dibujo científico le facilitaron su ingreso al Instituto de Botánica de la Universidad de Guadalajara en junio de 1985, por invitación de la maestra Luz María Villarreal de Puga y del profesor Jorge Alberto Pérez de la Rosa. Inmediatamente, se incorporó al trabajo con el proyecto Flora de Jalisco, iniciando así su carrera como botánico. También, en el Instituto de Botánica desarrolló su tesis titulada "Taxonomía, ecología y distribución del género Phaseolus (Leguminosae), en los municipios de Tlajomulco de Zúñiga y Jocotepec, Jalisco". Con este documento obtuvo el grado de Ingeniero Agrónomo en junio de 1991.

En 1991, la Universidad de Guadalajara inició un programa para el mejoramiento académico de su profesorado. Bajo este programa, Ray inició su maestría en la Facultad de Ciencias de la Universidad Nacional Autónoma de México en 1994. Terminó los créditos en junio de 1997, concluyó el trabajo de campo y redactó su tesis de posgrado sobre la Sistemática del complejo Vigna speciosa (Vigna sección Sigmoidotropis) para México, pero no obtuvo el grado. A su regreso a la Universidad de Guadalajara, retomó sus funciones como profesor en los programas de Agronomía y Biología.

Como profesor, Raymundo fue un referente en los cursos de Diversidad Vegetal, Espermatofitas, Vegetación y Flora de la Nueva Galicia, Colecciones Botánicas, Monocotiledóneas y Dicotiledóneas. En sus clases fue inspiración para muchos estudiantes. Sin embargo, lo mejor fueron sus excursiones botánicas. Su nombre automáticamente es asociado a la salida de campo a la costa de Jalisco. La excursión duraba cuatro días y se visitaba Ciudad Guzmán, El Grullo, Autlán de Navarro, Casimiro Castillo, La Huerta, Puerto Melaque, La Manzanilla y Chamela. A los estudiantes les mostraba la estructura y la composición del bosque de coníferas, el encinar, el bosque tropi- 
cal caducifolio, el bosque tropical subcaducifolio, el bosque mesófilo de montaña, el manglar y la vegetación en dunas costeras. Por supuesto, no podía faltar la visita a "El Botanero", un restaurant familiar, en el poblado de Casimiro Castillo. Acto seguido, la excursión se detenía en La Concha, municipio de La Huerta, para admirar petroglifos labrados en mármol.

Otra excursión típica de los cursos de Raymundo incluía a San Sebastián del Oeste y Puerto Vallarta. Después del arribo a San Sebastián, la excursión se dirigía a La Bufa. Al día siguiente, se continuaba hacia Puerto Vallarta siguiendo la trayectoria que en su tiempo siguiera Ynes Mexia. Su carácter afable, su amplio conocimiento de las plantas y su entera disposición para sus estudiantes lo convirtieron en uno de los profesores más populares de la Licenciatura en Biología. La influencia de Raymundo en la formación profesional de los estudiantes quedó manifestada en las tres generaciones de biólogos que tuvieron a bien ponerle su nombre. La lista sería muy larga si mencionamos todas las muestras de agradecimiento que recibió de otras generaciones de biólogos. Dirigió cinco tesis de licenciatura y fue el mentor de numerosos estudiantes de servicio social y prácticas profesionales.

Como investigador, Raymundo fue un botánico productivo. Sus grupos de interés fueron los géneros Phaseolus y Vigna (Fabaceae). También desarrolló idolatría por las familias Burseraceae y Anacardiaceae. Sus asociaciones vegetales favoritas fueron los bosques tropicales y el manglar. Para esos ecosistemas elaboró múltiples listados florísticos. Destacan los inventarios del Parque Nacional Isla Isabel, El Estero El Salado en Puerto Vallarta; La Manzanilla, municipio de La Huerta; La Yesca, barranca del Río Grande de Santiago; Cerro El Colli en Zapopan; Laguna de Sayula; Bahía de Banderas; Cerro Gordo, municipio de Arandas; Encarnación de Díaz y Área Natural Protegida Bosque El Nixticuil, San Esteban y El Diente. También participó en proyectos de manifestación de impacto ambiental como coordinador de flora. Publicó 22 artículos científicos incluidas las siguientes siete especies nuevas: Cypripedium luzmarianum R.González et Ramírez-Delgadillo (Orquidaceae), Bessera tuitensis RamírezDelgadillo (Themidiaceae), Polyanthes michoacana M.Cedano, Ramírez-Delgadillo et I.Enciso (Agavaceae), Phaseolus albescens McVaugh ex Ramírez-Delgadillo et A.Delgado (Fabaceae), Cleomella jaliscensis Villegas-Flores et Ramírez-Delgadillo (Capparaceae), Clethra fragrans L.M.González et Ramírez-Delgadillo (Clethraceae) y Agave rzedowskiana P.Carrillo, Vega et Ramírez-Delgadillo (Agavaceae). Sus proyectos y exploraciones botánicas fueron más numerosos en el occidente de México, pero también trabajó en otras partes del país. Sus 7,921 colectas botánicas están depositadas en el herbario IBUG donde fue curador en dos periodos. El primero comprendió de 1992 a 1994. El segundo inició en 1997 y continuó hasta su deceso.

En reconocimiento a su labor como botánico, le fueron dedicadas las siguientes especies: Bauhinia ramirezii Reynoso (Fabaceae), Deiregyne ramirezii R.González (Orchidaceae), Malaxis ramirezii R.González (Orchidaceae), Microthelys ramirezii R.González et Shalisko. (Orchidaceae, ined.), Bletia ramirezii R.González et CuevasFigueroa (Orchidaceae, ined.) y Potosia ramirezii R.González et L.Hernández H. (Orchidaceae, ined.). Con su partida, México ha perdido a uno de sus más entusiastas investigadores y formadores de botánicos.

Aarón Rodríguez Contreras y Leticia Hernández López

Herbario Luz María Villarreal de Puga del Instituto de Botánica

de la Universidad de Guadalajara

Departamento de Botánica y Zoología, Centro Universitario

de Ciencias Biológicas y Agropecuarias, Universidad de Guadalajara 